\title{
METODOLOGÍA DE CALIBRACIÓN DE DOS MODELOS DE SIMULACIÓN DEL TRANSPORTE DE AGUA EN EL SUELO EN CULTIVOS HORTÍCOLAS
}

Sánchez de Oleo, C. ${ }^{1}$, Jaramillo, C. ${ }^{2}$, Illera, I. ${ }^{3}$, Lidón, A. ${ }^{4}$, Ramos, C. ${ }^{5}$, Ginestar, $D .{ }^{6}$

1 Profesor, Escuela de Física, Universidad Autónoma de Santo Domingo, C/ Alma Mater, Santo Domingo, República Dominicana, matematicacarlo@hotmail.com

${ }^{2}$ Doctoranda Dpto. Producción Vegetal, Universitat Politècnica de València, Camino de Vera S/N 46022 Valencia, ilem750128@hotmail.com

${ }^{3}$ Estudiante, ETSICC, Universitat Politècnica de València, Camino de Vera S/N 46022 Valencia, ivilgme@cam.upv.es

${ }^{4}$ Profesor, Grupo Re-Forest, Departamento de Ingeniería Hidráulica y Medio Ambiente. Universitat Politècnica de València. Camino de Vera, S/N 46022 Valencia, alidon@qim.upv.es

5 Investigador, Instituto Valenciano de Investigaciones Agrarias, Apdo. Oficial, 46113 Moncada (Valencia), ramos carmon@gva.es

${ }^{6}$ Profesor, Instituto de Matemática Multidisciplinar. Universitat Politècnica de València. Camino de Vera, S/N 46022 Valencia, dginesta@mat.upv.es

\section{Resumen}

El uso de modelos de simulación de la dinámica del agua en el suelo requiere del conocimiento de un gran número de parámetros, algunos de los cuales son difíciles de medir y es necesaria su calibración a partir de medidas experimentales. Por ello, es conveniente elegir aquellos parámetros que mayor incidencia tienen en los procesos a estudiar, simplificando con ello el proceso de calibración del modelo. En este trabajo se propone la utilización de métodos de análisis de sensibilidad global para determinar los parámetros más influyentes en los errores de la predicción del contenido de humedad del suelo de dos modelos de simulación con diferente grado de complejidad, LEACHM y EU-Rotate_N. Se ha hecho uso de los valores medidos de la humedad del suelo en dos ensayos de cultivo de coliflor en dos años consecutivos, utilizando los datos del primer ensayo para la calibración de ambos modelos y los datos del segundo ensayo para evaluar la capacidad predictiva de los mismos una vez calibrados. Los resultados muestran que es posible obtener buenas predicciones calibrando solo un número reducido de parámetros.

\section{1- Introducción}

Los modelos de simulación que describen el comportamiento del agua y nitrógeno en el suelo son herramientas útiles para planificar estrategias de manejo del riego y la fertilización, de forma que sin mermas de la producción se minimicen algunos de los problemas ambientales derivados de estas prácticas agrícolas (Cannavo et al. 2008; Kersebaum et al. 2007). El uso de estos modelos de simulación requiere del conocimiento de un gran número de parámetros, algunos de los cuales son difíciles de medir y es necesaria su calibración a partir de medidas 
experimentales (Jung et al. 2010) y, probablemente, esta es la razón por la cual los modelos de simulación se utilizan raramente como herramienta de apoyo a la gestión del riego y otras prácticas agrícolas como la fertilización. Además, es conocido que los modelos con un gran número de parámetros variables, pueden presentar problemas de sobreparametrizacion y sobreajuste de los datos (Makowski et al. 2006).

Por otra parte, la calibración de modelos con un elevado número de parámetros requiere de un gran número de medidas experimentales que en ocasiones son difíciles y costosas de obtener a partir de experimentos de campo. Por ello, es conveniente elegir aquellos parámetros que mayor incidencia tienen en los procesos a estudiar, simplificando con ello el proceso de calibración del modelo. El objetivo de este trabajo es proponer un análisis de sensibilidad de dos modelos que simulan el transporte de agua en sistemas agrícolas, y su utilización para la calibración de estos modelos a partir de medidas del contenido de agua en el suelo obtenidas en parcelas de coliflor cultivadas en dos años consecutivos.

\section{2- Material y métodos}

Los datos utilizados para calibrar los dos modelos se obtuvieron de un experimento llevado a cabo en dos plantaciones comerciales de coliflor en Paterna (Valencia, 39²9'32,12" N y 0²6'21,19” W, 14 metros sobre el nivel del mar). El suelo es profundo sin piedras, de textura franco arcillosa en las dos primeras capas (0-15 cm y $15-30 \mathrm{~cm})$ y arcillo-limosa en la tercera capa considerada en el ensayo $(30-45 \mathrm{~cm})$. La materia orgánica variaba entre $35 \mathrm{~g} \mathrm{~kg}^{-1}$ en la capa superficial y $16 \mathrm{~g}$ $\mathrm{kg}^{-1}$ en las capas más profundas. La densidad aparente del suelo osciló entre 1128 $\mathrm{kg} \mathrm{m}^{-3}$ en la primera capa y $1600 \mathrm{~kg} \mathrm{~m}^{-3}$ en la tercera capa. En la Tabla 1 se recogen las principales características del suelo de las dos parcelas del ensayo.

Tabla 1. Propiedades del suelo en las dos parcelas del ensayo.

\begin{tabular}{lccc}
\hline & \multicolumn{3}{c}{ Profundidad $(\mathrm{cm})$} \\
\cline { 2 - 4 } Propiedades & $0-15$ & $15-30$ & $30-45$ \\
\hline Textura (\%) & 67 & 35 & 24 \\
\multicolumn{1}{c}{ Arena } & 17 & 35 & 39 \\
Limo & 16 & 30 & 37 \\
Arcilla & Franco & Franco & Franco \\
Clase textural (USDA) & arenoso & arcilloso & arcilloso \\
Carbono orgánico $\left(\mathrm{g} \mathrm{C} \mathrm{kg}^{-1}\right)$ & 9.0 & 8.0 & 8.0 \\
Nitrógeno orgánico $\left(\mathrm{g} \mathrm{N} \mathrm{kg}^{-1}\right)$ & 1.0 & 0.9 & 0.9 \\
pH (KCl) & 7.5 & 7.5 & 7.5 \\
Capacidad intercambio catiónico $\left(\mathrm{cmol}_{\mathrm{c}} \mathrm{kg}^{-1}\right)$ & 8.8 & 12.1 & - \\
Densidad aparente $\left(\mathrm{Mg} \mathrm{m}^{-3}\right)$ & 1.29 & 1.60 & 1.72 \\
\hline
\end{tabular}

La coliflor se trasplantó el 17 de septiembre de 2012 en la parcela 1 y el 15 de septiembre de 2013 en la parcela 2. La distancia entre filas fue de $0.64 \mathrm{~m}$ y la 
separación entre plantas de $0.67 \mathrm{~m}$. La coliflor se cosechó el 20 de febrero de 2013 en la parcela 1 y el 15 de febrero de 2014 en la parcela 2. El riego fue por surcos utilizando agua del río Turia y la lámina de agua aplicada fue medida en cada riego. La lluvia se registró en una estación meteorológica situada cerca de las parcelas. En cada parcela se tomaron muestras de suelo a tres profundidades coincidentes con las capas de suelo anteriormente mencionadas. Los muestreos tuvieron una periodicidad de 15-21 días dependiendo de la época, y se prolongaron hasta el inicio del siguiente cultivo. En cada muestra se determinó, entre otras variables, la humedad del suelo mediante secado del suelo a $105^{\circ} \mathrm{C}$.

Para simular la dinámica de agua se utilizaron dos modelos diferentes implementados en los códigos LEACHM y EU-Rotate_N. El modelo del código LEACHM (Wagenet \& Hutson, 1989) es un modelo que describe el régimen de agua y transporte de solutos en suelos no saturados o parcialmente saturados. La dinámica del agua se obtiene de la resolución de la ecuación de Richards y de las ecuaciones de Campbell para establecer las relaciones entre la humedad, el potencial matricial y la conductividad hidráulica del suelo. La calibración de este modelo implica la determinación de los parámetros a y $b$ de Campbell así como de la conductividad hidráulica saturada del suelo, Khsat, en cada una de las capas de suelo consideradas.

El código EU-Rotate_N (Rahn et al. 2007) se ha desarrollado en el marco de un proyecto europeo y consiste en un conjunto de módulos que simulan el crecimiento de las plantas, la dinámica del nitrógeno y del agua en el suelo. Para describir la dinámica del agua se utiliza un modelo compartimental de tipo "tipping bucket", que hace uso de un menor número de parámetros que los modelos de tipo físico. Los parámetros básicos del modelo son el contenido de agua a capacidad de campo (FC), a saturación (SAT), en el punto de marchitez permanente (PWP) y los coeficientes de drenaje (CD), que determinan la transferencia de agua entre las distintas capas. La evapotranspiración del cultivo se calcula con el método FAO (Allen et al. 1998), siendo necesario disponer de la evapotranspiración de referencia (ETo) y los correspondientes coeficientes de cultivo.

Para reducir el número de parámetros a calibrar en cada uno de los modelos se ha tomado como referencia las medidas experimentales de humedad volumétrica en cada una de las capas de suelo consideradas y se ha construido una función que mide el error de la predicción de cada uno de los modelos:

$$
\varepsilon=\sum_{i=1}^{n c a p a s} \frac{\left(\sum_{j=1}^{n m e d}\left(\theta_{v i j}-\theta_{v i j}^{*}\right)^{2}\right)^{1 / 2}}{\left(\sum_{j=1}^{n m e d}\left(\theta_{v i j}\right)^{2}\right)^{1 / 2}}
$$

donde ncapas es el número de capas de suelo consideradas (tres en este caso), $n$ med es el número de medias de humedad en cada capa, $\theta_{v i j}$ es la medida j-ésima de la humedad volumétrica en la capa i-ésima y $\theta_{v i j}^{*}$ es el valor j-ésimo de la humedad volumétrica en la capa i-ésima predicho por el modelo. Utilizando este valor del error se ha hecho un análisis de sensibilidad global de los parámetros hidráulicos de ambos modelos, determinando aquellos parámetros que más influyen en la variabilidad del error. Se ha comparado el funcionamiento de dos métodos de 
análisis de sensibilidad. El método LH-OAT (Jung et al. 2010) y el método FAST (Cannavó, 2012). En el primero de ellos se consideran $p$ parámetros para los que se dispone de un valor máximo y mínimo, y cada intervalo se divide en $N$ subintervalos. Se realiza un muestreo mediante el método del hipercubo latino y en cada punto del muestreo se varían de uno en uno los $p$ parámetros, construyendo el índice de sensibilidad $l_{i}$ para cada parámetro $e_{i}$ calculado como:

$$
I_{i}=\sum_{j=1}^{N} \frac{\left|M\left(e_{1 j}, \cdots, e_{i j}\left(1+f_{i}\right), \cdots, e_{p j}\right)-M\left(e_{1 j}, \cdots, e_{i j}, \cdots, e_{p j}\right)\right|}{N \times f_{i} \times M\left(e_{1 j}, \cdots, e_{i j}, \cdots, e_{p j}\right)},
$$

donde $M()$ hace referencia a la función que define el modelo, $f_{i}$ es la fracción de cambio introducida en el parámetro $i$ y $j$ hace referencia a un punto del muestreo.

El método FAST (Fourier Amplitude Sensitivity Test) (Cukier et al. 1973) se basa en una descomposición de la varianza, de forma que se cuantifica la contribución de cada uno de los parámetros del modelo a la varianza total. Si $Y=M\left(e_{1}, \cdots, e_{p}\right)$ es la función que define el modelo en estudio, el método FAST estima los índices de sensibilidad de primer orden como

$$
S_{i}=\frac{\operatorname{Var}\left(E\left(Y \mid e_{i}\right)\right)}{\operatorname{Var}(Y)},
$$

donde $E\left(Y \mid e_{i}\right)$ es el valor esperado del resultado del modelo si el parámetro $e_{i}$ se fija a un valor dado. Para evaluar estos índices de sensibilidad de primer orden se han de calcular integrales múltiples. El método FAST realiza un muestreo en el espacio de parámetros y una transformación de los mismos que permite reducir las integrales múltiples a integrales unidimensionales utilizando las series de Fourier. Los detalles del método pueden ser consultados en Saltelli et al. (1999). Los cálculos realizados en este trabajo se han obtenido utilizando la librería GSAT que se describe en Cannavó (2012).

Los parámetros seleccionados para realizar el análisis de sensibilidad de cada uno de los modelos y su rango de variación estimado se presentan en la Tabla 2. Los valores del resto de parámetros de ambos modelos se fijaron al valor por defecto de cada uno de los códigos.

Una vez identificados los parámetros más sensibles de los modelos, se procedió a su calibración, dejando el resto de parámetros con el valor medio del rango de variación considerado. La calibración se llevó a cabo obteniendo los valores de los parámetros que hacen mínima la función de error (1) mediante el método simplex de Nelder-Mead (Lagarias et al. 1998). En concreto se utilizó la función fminsearch del paquete MATLAB. La bondad del proceso de calibración se ha medido utilizando los estadísticos diferencia media, RMSE, NRMSE y coeficiente de correlación de la regresión de los valores medidos frente a los predichos por el modelo (Lidón et al. 2013). 
Tabla 2. Parámetros hidráulicos evaluados en cada uno de los modelos.

\begin{tabular}{lcccc}
\hline \multicolumn{1}{c}{ Parámetro } & Símbolo & Unidades & $\begin{array}{c}\text { Valor } \\
\text { inferior }\end{array}$ & $\begin{array}{c}\text { Valor } \\
\text { superior }\end{array}$ \\
\hline Modelo LEACHM & & & & \\
Parámetro a ec. Campbell capa 0-15 & a1 & $\mathrm{kPa}$ & -0.149 & -10.000 \\
Exponente $b$ ec. Campbell capa 0-15 & $\mathrm{b} 1$ & - & 0.140 & 10.000 \\
Conductividad hidráulica saturada capa 0-15 & $\mathrm{k} 1$ & $\mathrm{~mm} / \mathrm{día}$ & 264 & 13400 \\
Parámetro a ec. Campbell capa 15-30 & $\mathrm{a} 2$ & $\mathrm{kPa}$ & -0.149 & -10.000 \\
Exponente $b$ ec. Campbell capa 15-30 & $\mathrm{b} 2$ & - & 0.140 & 20.000 \\
Conductividad hidráulica saturada capa 15-30 & $\mathrm{k} 2$ & $\mathrm{~mm} / \mathrm{día}$ & 264 & 13400 \\
Parámetro a ec. Campbell capa 30-45 & a3 & $\mathrm{kPa}$ & -0.149 & -10.000 \\
Exponente b ec. Campbell capa 30-45 & b3 & - & 0.140 & 25.000 \\
Conductividad hidráulica saturada capa 30-45 & $\mathrm{k} 3$ & $\mathrm{~mm} / \mathrm{dí}^{2}$ & 264 & 13400 \\
& & & & \\
Modelo EU-Rotate_N $N$ & & & & \\
Humedad a capacidad de campo capa 0-15 & FC1 & $\mathrm{cm}^{3} / \mathrm{cm}^{3}$ & 0.150 & 0.350 \\
Humedad de saturación capa 0-15 & SAT1 & $\mathrm{cm}^{3} / \mathrm{cm}^{3}$ & 0.400 & 0.550 \\
Coeficiente de drenaje capa 0-15 & CD1 & - & 0.100 & 1.000 \\
Humedad a capacidad de campo capa 15-30 & FC2 & $\mathrm{cm}^{3} / \mathrm{cm}^{3}$ & 0.150 & 0.300 \\
Humedad de saturación capa 15-30 & SAT2 & $\mathrm{cm}^{3} / \mathrm{cm}^{3}$ & 0.400 & 0.470 \\
Coeficiente de drenaje capa 15-30 & CD2 & - & 0.100 & 1.000 \\
Humedad a capacidad de campo capa 30-45 & FC3 & $\mathrm{cm}^{3} / \mathrm{cm}^{3}$ & 0.150 & 0.300 \\
Humedad de saturación capa 30-45 & SAT3 & $\mathrm{cm}^{3} / \mathrm{cm}^{3}$ & 0.350 & 0.400 \\
Coeficiente de drenaje capa 30-45 & CD3 & - & 0.100 & 1.000 \\
\hline
\end{tabular}

\section{3- Resultados y discusión}

Se han estudiado los índices de sensibilidad de la función error (1) respecto de los parámetros mostrados en la Tabla 2. Para el método LH-OAT se han obtenido los índices de sensibilidad usando 50 niveles en el intervalo de variación de los parámetros y 5 repeticiones. En las Figuras 1 y 2 se muestra la media de los índices obtenidos junto con la barra de error asociada a cada desviación típica. En estas Figuras también se muestran los índices de sensibilidad de primer orden para los distintos parámetros de los modelos calculados mediante el método FAST.
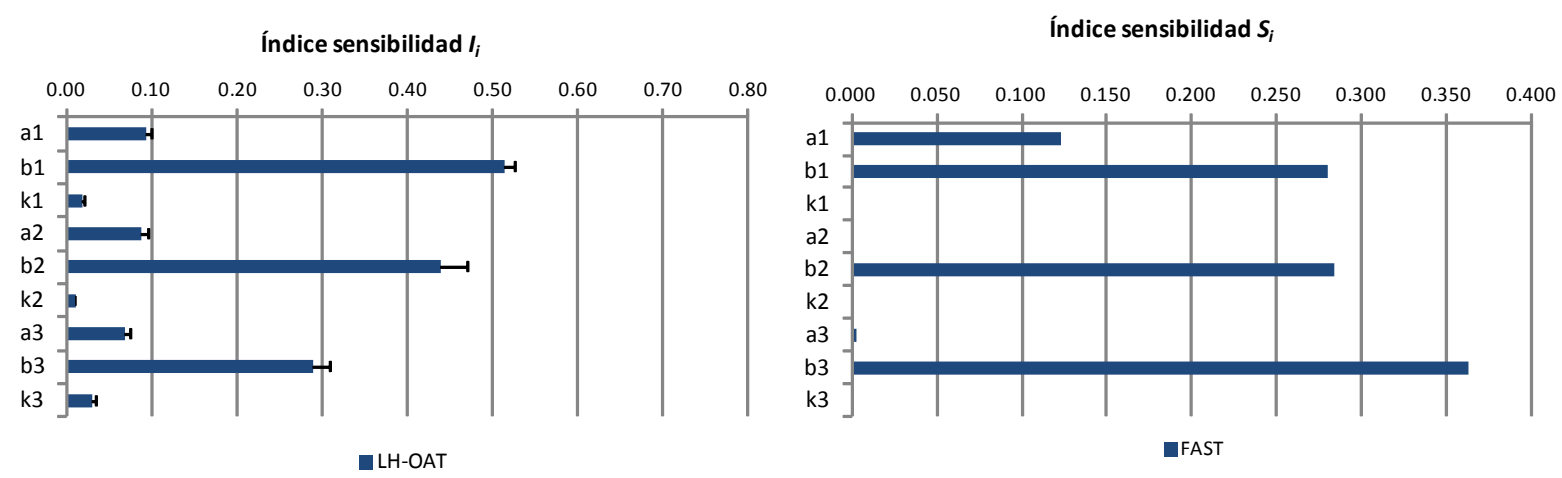

Figura 1. Índices de sensibilidad de los parámetros hidráulicos del modelo LEACHM obtenidos mediante LH-OAT y FAST. 

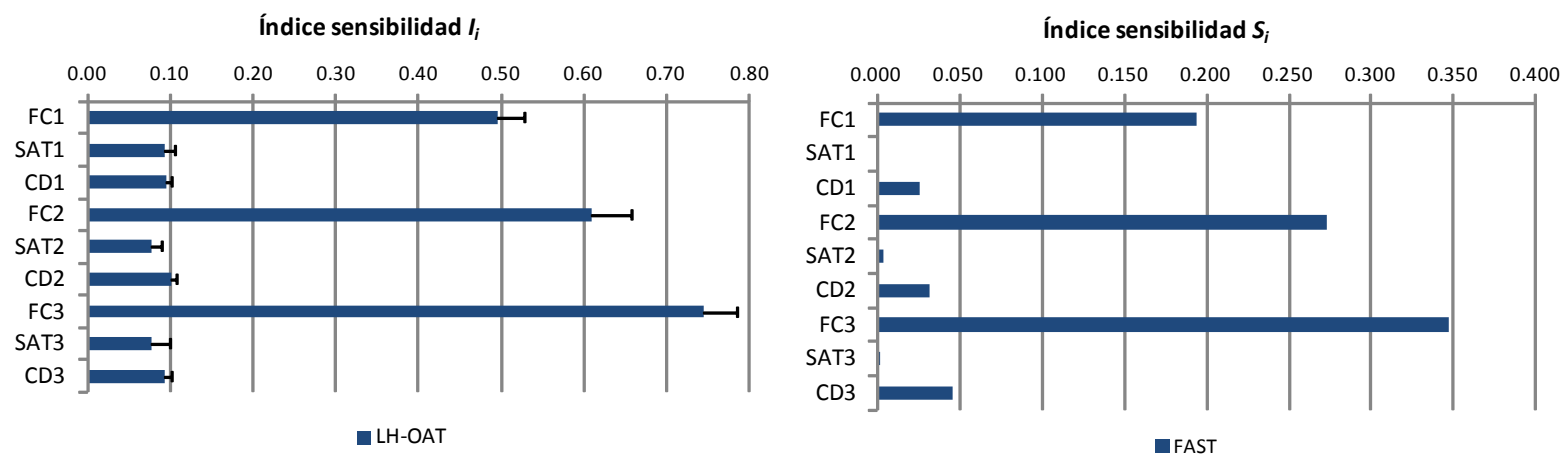

Figura 2. Índices de sensibilidad de los parámetros hidráulicos del modelo EU-Rotate_N obtenidos mediante LH-OAT y FAST.

Para el modelo LEACHM ambos análisis de sensibilidad muestran que los parámetros que más afectan al error en la predicción del contenido de agua en el suelo son el exponente de la ecuación de Campbell para cada una de las capas consideradas, mientras que para el modelo EU-Rotate_N los parámetros más influyentes son la humedad a capacidad de campo de cada una de las capas de suelo. Por tanto, éstos son los parámetros seleccionados para el proceso de calibración de los modelos. En la Tabla 3 se muestran los valores iniciales y los valores obtenidos tras el proceso de calibración para los parámetros seleccionados para cada modelo. Los estadísticos obtenidos para la calibración de la lámina de agua calculada con los dos modelos (Tabla 4) muestran que el ajuste obtenido para ambos modelos fue bueno, dado el bajo valor de NRMSE y la baja diferencia media obtenida en cada modelo.

Tabla 3. Parámetros calibrados en cada modelo.

\begin{tabular}{ccccc}
\hline Modelo & Parámetro & & $\begin{array}{c}\text { Valor } \\
\text { inicial }\end{array}$ & $\begin{array}{c}\text { Valor } \\
\text { calibrado }\end{array}$ \\
\hline LEACHM & b1 & & 5.138 & 5.512 \\
& b2 & & 6.138 & 9.876 \\
EU-Rotate_N & b3 & & 7.000 & 19.357 \\
& FC1 & $\mathrm{cm}^{3} / \mathrm{cm}^{3}$ & 0.250 & 0.362 \\
& FC2 & $\mathrm{cm}^{3} / \mathrm{cm}^{3}$ & 0.270 & 0.263 \\
& FC3 & $\mathrm{cm}^{3} / \mathrm{cm}^{3}$ & 0.250 & 0.299 \\
\hline
\end{tabular}

Tabla 4. Estadísticos de la calibración de los modelos LEACHM y EU-Rotate_N.

\begin{tabular}{ccc}
\hline & \multicolumn{2}{c}{ Lámina $(\mathrm{mm})$ 0-45 $\mathrm{cm}$} \\
\cline { 2 - 3 } & LEACHM & EU-Rotate_N \\
\hline Diferencia media & -2.8 & 1.5 \\
RMSE & 12.5 & 13.2 \\
NRMSE & 0.10 & 0.11 \\
$r$ & 0.727 & 0.888 \\
\hline
\end{tabular}

Para evaluar la capacidad predictiva de los modelos una vez calibrados, se fijaron los valores de los parámetros calibrados con los datos del ensayo 1 y se 
predijo la lámina de agua y la humedad volumétrica en cada capa para el ensayo 2 en la segunda parcela. En la Figura 3 se muestran los valores predichos por ambos modelos y los medidos a lo largo del ensayo, y en la Tabla 5 se recogen los estadísticos obtenidos en la predicción de la lámina de agua. Se observa como utilizando sólo tres parámetros en el proceso de calibración, se obtienen resultados con una precisión adecuada para utilizar los modelos como herramientas predictivas en situaciones similares a las de la calibración.

Tabla 5. Estadísticos de la predicción de los modelos LEACHM y EU-Rotate_N para el ensayo 2.

\begin{tabular}{ccc}
\hline & \multicolumn{2}{c}{ Lámina $(\mathrm{mm})$ 0-45 cm } \\
\cline { 2 - 3 } & LEACHM & EU-Rotate_N \\
\hline Diferencia media & 0.9 & 3.0 \\
RMSE & 8.3 & 7.8 \\
NRMSE & 0.07 & 0.07 \\
$r$ & 0.686 & 0.799 \\
\hline
\end{tabular}

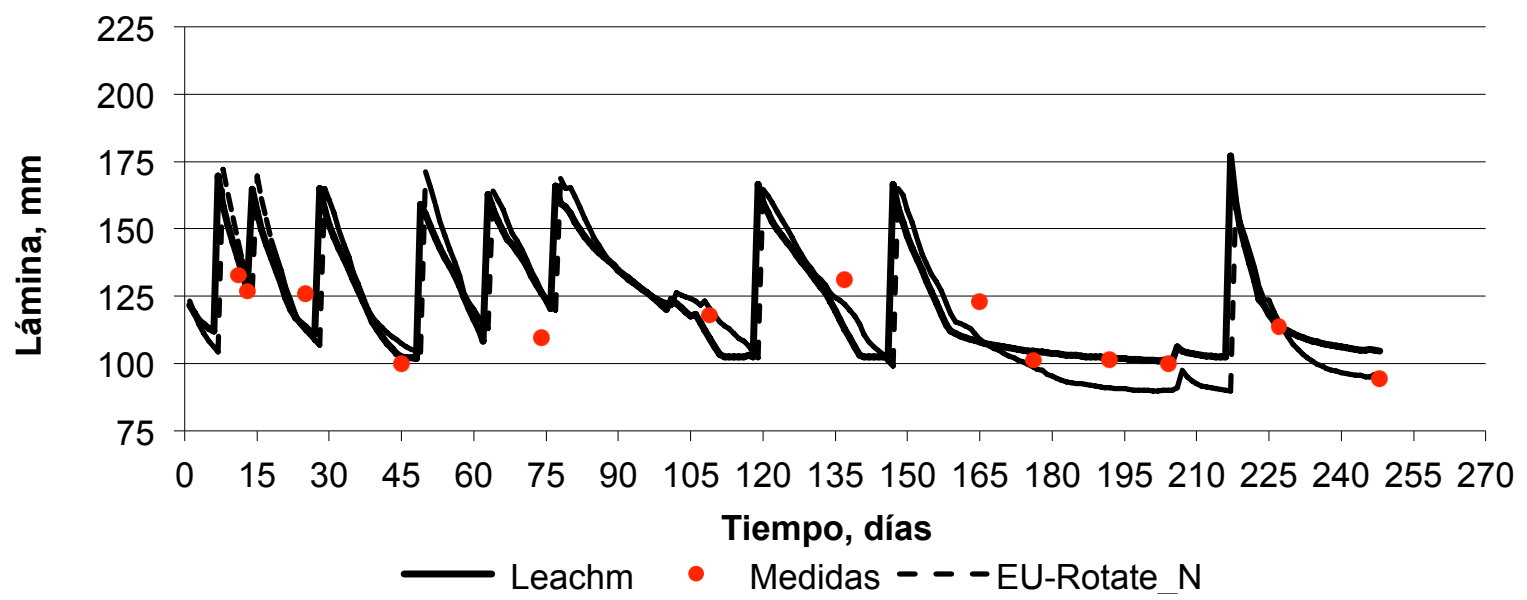

Figura 3. Lámina de agua simulada y medida en el perfil del suelo $(0-45 \mathrm{~cm})$ a lo largo del cultivo de coliflor correspondiente al ensayo 2 .

\section{4- Conclusiones}

Los modelos de simulación de la dinámica del agua en el suelo presentan un gran número de parámetros que es necesario ajustar a partir de medidas experimentales que no siempre son fáciles de obtener. Es necesario disponer de metodologías que permitan reducir el número de parámetros a ajustar para evitar la sobreparametrización del modelo. En este trabajo se ha propuesto la utilización de índices de sensibilidad global de los modelos utilizando como variable el error cuadrático medio entre los valores predichos y medidos para determinar los parámetros más sensibles. Estos parámetros se han utilizado en un proceso de calibración para determinar la humedad del suelo en una parcela cultivada con coliflor. Los dos métodos de sensibilidad utilizados han dado como resultado los mismos parámetros dominantes que, una vez calibrados, han permitido predecir con una precisión razonable el contenido de agua en el suelo, tanto para en el ensayo de 
campo utilizado para calibrar el modelo como en el ensayo utilizado para evaluar la capacidad predictiva de los mismos. Esto posibilita la utilización de estos modelos para mejorar las recomendaciones de manejo del agua en parcelas bajo regadío.

\section{5- Agradecimientos}

Este trabajo ha sido subvencionado parcialmente por el Ministerio de Economía y Competitividad mediante el proyecto INIA-RTA 2011-00136-C04-01.

\section{6- Bibliografía}

Allen, R.G., Pereira L.S., Raes D., Smith M. (1998). Crop evapotranspiration. Guidelines for computing crop water requirements. FAO Irrigation and Drainage Paper $n^{\circ} 56, F A O$, Roma.

Cannavó F. (2012). Sensitivity analysis for volcanic source modeling quality assessment and model selection. Computers \& Geosciences, 44, 52-59.

Cannavo, P., Recous, S., Parnaudeau, V., Reau, R. (2008). Modeling N dynamics to assess environmental impacts of cropped soils. Advances in Agronomy 97, 131-174.

Cukier, R.I., Fortuin, C.M., Shuler, K.E., Petschek, A.G., Schaibly, J.H. (1973). Study of the sensitivity of coupled reaction systems to uncertainties in rate coefficients. I theory. The Journal of Chemical Physics 59, 3873-3878.

Jung, Y.W., Oh, D.S., Kim, M., Park, J.W. (2010). Calibration of LEACHN model using LHOAT sensitivity analysis. Nutr. Cycl. Agroecosyst. 87, 261-275.

Kersebaum, K.C., Hecker, J.M., Mirschel, W., Wegehenkel, M. (2007). Modelling water and nutrient dynamics in soil-crop systems: a comparison of simulation models applied on common data sets. In: Kersebaum, K.C., Hecker, J.M., Mirschel, W., Wegehenkel, M. (Eds.), Modelling Water and Nutrient Dynamics in Soil-Crop Systems. Springer, Dordrecht, The Netherlands, pp. 1-17.

Lagarias, J.C., Reeds J.A., Wright M.H., Wright P.E. (1998). Convergence Properties of the Nelder-Mead Simplex Method in Low Dimensions. SIAM Journal of Optimization, 9, $112-147$.

Lidón, A., Ramos, C., Ginestar, D., Contreras, W. (2013). Assessment of LEACHN and a simple compartmental model to simulate nitrogen dynamics in citrus orchards. Agricultural Water Management, 121, 42-53.

Makowski, D., Hillier, J., Wallach, B., Andrieu, B., Jeuffroy, M.H. (2006). Parameter estimation for crop models. In: D. Wallach et al. (eds.): Working with dynamic crop models. Amsterdam. Elsevier, 101-149.

Rahn, C., Zhang, K., Lillywhite, R., Ramos, C., Doltra, J., De Paz, J. M., Riley, H., Fink, M., Nendel, C., Thorup Kristensen, K., Pedersen, A., Piro, F., Venezia, A., Firth, C., Schmutz, U., Rayns, F., Strohmeyer, K. (2010). EU-Rotate_N-a decision support system-to predict environmental and economic consequences of the management of nitrogen fertiliser in crop rotations. European Journal of Horticultural Science, 75, 2032.

Saltelli, A., Tarantola, S., Chan, K.S. (1999). A quantitative model-independent method for global sensitivity analysis of model output. Technometrics, 41, 39-56.

Wagenet, R.J., Hutson, J.L. (1989). LEACHM: Leaching Estimation and Chemistry Model: A Process Based Model of Water and Solute Movement, Transformations, Plant Uptake and Chemical Reactions in the Unsaturated Zone. Ver. 2. Water Resour. Inst. Cornell University, Ithaca, New York. 